\title{
LA LECTURE DU GESTE, UN OUTIL POUR LA RECHERCHE EN DANSE ${ }^{1}$
}

\author{
Christine Roquet \\ Professeure à l'Université Paris 8 - Département de Danse \\ Email: chroquet@free.fr
}

A partir de questionnements sur le corps, cette conférence expliquera comment, au sein des études en danse en France, l'analyse du mouvement considère que c'est le geste qui fabrique le «corps ». Après l'exposé de la théorie des quatre structures de la corporéité de Hubert Godard, nous démontrerons l'intérêt qu'il peut avoir à faire de la lecture du geste un outil pour la recherche en danse. Comment passer de la marche à la danse ? Quel dialogue entre les interprètes d'un duo amoureux ? Des exemples concrets d'analyse pris dans la comédie musicale The band wagon (Vicente Minelli, 1953, avec Fred Astaire et Cyd Charisse) viendront illustrer nos propos.

\section{Mots-clefs}

Analyse du Mouvemente. Geste. Corps. Lecture du Geste.

\begin{abstract}
A partir de questionamentos sobre o corpo, esta conferência explicará como, no contexto dos estudos em dança na França, a análise do movimento considera que é o gesto que fabrica o "corpo". Após expor a teoria das quatro estruturas da corporeidade, de Hubert Godard, demonstraremos a importância de fazer da leitura do gesto uma ferramenta para a pesquisa em dança. Como passar da caminhada à dança? Qual o diálogo entre os intérpretes de um duo amoroso? Exemplos concretos de análise, retirados da comédia musical $A$ Roda da Fortuna (Vincent Minelli, 1953, com Fred Astaire e Cyd Charisse) virão ilustrar nossas proposições.
\end{abstract}

\section{Palavras-chave}

Análise do Movimento. Gesto. Corpo. Leitura do Gesto.

1 Conférence d'ouverture. I Colóquio Nacional Artes do Movimento: A Análise do Movimento e a Pesquisa em Artes - organisé par le Groupe de Recherche Artes do Movimento, 12 et 13 mars de 2015, École de Théâtre du Centre de Lettres et Arts de UNIRIO. Ce colloque a été intégré dans le cours Approche Systémique du Geste Expressif (45h), avec Christine Roquet, au PPGAC à I'Université UNIRIO. 


\section{Introduction}

Permettez-moi tout d'abord de vous remercier de m'accueillir parmi vous

à l'occasion de ce colloque'. Depuis mon premier séjour à Rio de Janeiro en 2011 chaque visite au Brésil est un plaisir renouvelé. Ce séjour inaugural de 2011 à Unirio a été le départ d'un travail conjoint avec des personnes dont les qualités d'écoute et d'accueil sont pour moi un émerveillement. De ma visite à Unirio en 2011, nous avons tiré une conférence appelée «De l'analyse du mouvement à l'approche systémique du geste expressif » qui est éditée dans les Annales du I Seminário Internacional Corpo Cênico: Linguagens e Pedagogias². Je vais vous rappeler ce qui est dit dans cette conférence :

- Dans un premier temps, j'y explique pourquoi il convient de parler davantage de geste que de mouvement. Nous pourrions synthétiser cela avec Hubert Godard, qui propose de « distinguer le mouvement, compris comme un phénomène relatant les stricts déplacements des différents segments du corps dans l'espace - au même titre qu'une machine produit un mouvement - et le geste, qui s'inscrit dans l'écart entre ce mouvement et la toile de fond tonique et gravitaire du sujet : c'est-à-dire le pré-mouvement dans toutes ses dimensions affectives et projectives. C'est là que réside l'expressivité du geste humain, dont est démunie la machine. $»^{3}$

1 Colóquio Nacional Artes do Movimento: A Análise do Movimento e a Pesquisa em Artes.

2 En ligne dans la revue Percevejo online : http://www.seer. unirio.br/index.php/opercevejoonline/article/view/1784/1447. Le DVD des annales est disponible en ligne : https://player. vimeo.com/video/38588940.

3 « Le geste et sa perception » postface à Isabelle Ginot et Marcelle Michel, La danse au XXème siècle, Paris, Bordas, 1995, p. 225.
- Dans un deuxième temps, j'explore les enjeux et les limites de certaines tentatives de lecture du geste, en particulier les approches sémiologiques, insuffisantes selon nous pour penser le sens du geste en deçà du signe.

- Dans un troisième temps, j'explique comment en France s'organise la « discipline » ou plutôt le champ d'interrogations que j'appelle « analyse du mouvement » et, plus explicitement, j'expose les enjeux du travail d'Hubert Godard à Paris 8, que l'on pourrait appeler « approche complexe du geste expressif ».

Il existe donc déjà des écrits en portugais à propos de notre travail ${ }^{4}$, auxquels il faut ajouter, et cela est d'une grande importance, le travail d'atelier traversé ensemble à Rio de Janeiro, Fortaleza, Porto Alegre, Salvador... La pratique est en effet indispensable à la compréhension des outils utilisés en analyse du mouvement, quelle qu'elle soit. AFCMD ${ }^{5}$, Effort ou Bartenieff Fundamentals... seul le passage par l'expérience permet de saisir la subtilité de notions qui ont toutes émergé de la pratique du geste et de sa perception. Aussi nous avons expérimenté, en atelier, différentes manières de marcher, nous avons pu saisir comment le coloris ${ }^{6}$

4 De mon côté, en plus de la conférence déjà citée Christine Roquet, Análise do movimento e análise de obras coreográficas: o corpo cênico entre a dança e o teatro. São Paulo: Annablume, 2013, p. 249-255.

Ao encontro da criação: a análise do movimento e o processo de criação coreográfica, in: ABRACE, Da Cena Contemporânea. Porto Alegre, Brazil, p. 39-45.

Da análise do movimento à abordagem sistêmica do gesto expressivo, Tradução: Joana Ribeiro e Marito Olsson-Forsberg. Rio de Janeiro, O Percevejo online, Dossiê Corpo Cênico - linguagens e pedagogias, vol. 3, n 1, PPGAC/ UNIRIO, 2011. p.01-15.

Corps, geste, corporéité... penser depuis la danse, conférence d'ouverture du colloque Seminário Dança, Teatro, Educação. O corpo do corpo no corpo. Université Fédérale du Ceará, Fortaleza, août 2014, à paraitre.

5 Analyse Fonctionnelle du Corps dans le Mouvement Dansé.

6 La couleur sort du tube du peintre alors que le coloris est ce qu'il en fait. 
du geste pouvait nuancer différemment la figure chorégraphique et nous avons observé les écarts entre deux modes différents d'opérer du geste dansé avec la lecture du duo "The babitt and the bromide ${ }^{7}$ ». C'est avec tout cela en poche que nous pouvons aujourd'hui aborder notre thématique : la lecture du geste, un outil pour la recherche en danse.

II n'y a pas de corps...

Mais en ouverture de ce colloque je voudrais déjà présenter les fondements de ce travail entrepris par Godard et moi-même. Pour ce faire, je voudrais partir d'une provocation, qui peut paraître tout à fait incongrue pour ce pays singulier qu'est le Brésil, cette provocation est la suivante : « il n'y a pas de corps ». Les européens ne manquent pourtant pas de mots pour vanter les « corps libérés » des cariocas sur la plage de Copacabana et nous faisons du Brésil l'exemple même d'une culture « du corps » (réduit ici à ses apparences). Mais, contrairement aux idées toutes faites je suis certaine que les danseurs et danseuses brésiliens auront compris, à la fin de cette conférence, pourquoi j'ai commencé par cette provocation.

\section{Le poids, une force}

Vous me direz, « tout de même, si Fred Astaire est si léger c'est bien parce qu'il a un corps fin et longiligne "... Je voudrais que nous commencions par là, par cette question de la morphologie. En faisant ma thèse sur Roméo et Juliette j'ai été amenée à comparer souvent

7 Extrait du film Ziegfeld follies de Vicente Minelli (1946). différentes versions de la même œuvre (version MacMillan ou Preljocaj). En regardant deux versions du ballet de MacMillan, l'une dansée par Margot Fonteyn (1965).

Figure 1 - R. Nouréev et M. Fonteyn dans Roméo et Juliette de K. MacMillan en 1965

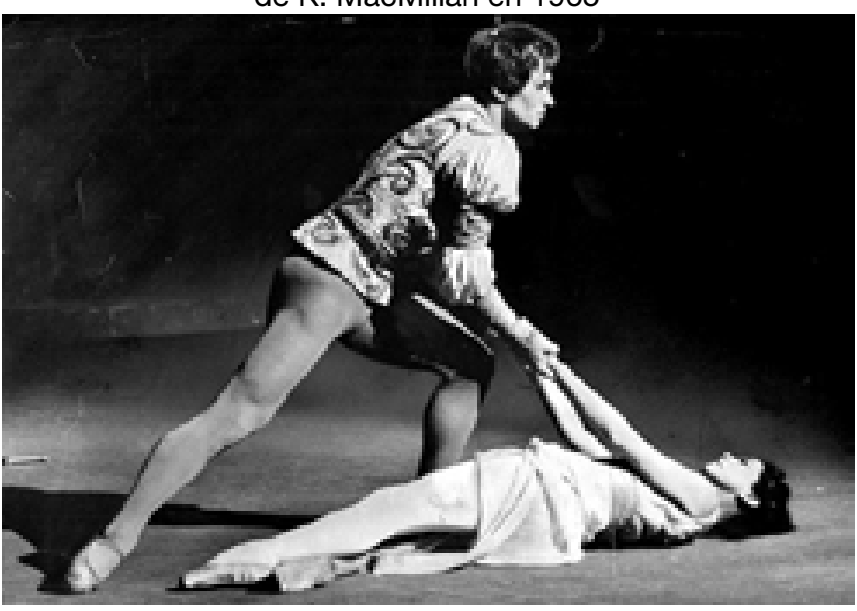

Source : New York Times (2007). ${ }^{8}$

et l'autre par Allessandra Ferri (1984).

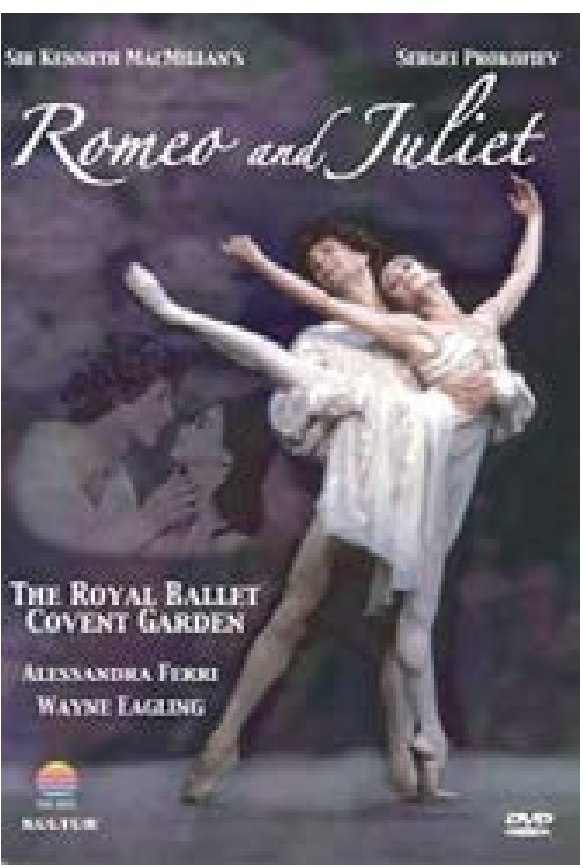

Source : Met Opera Shop (2017)

8 Photographie téléchargée sur : http://www.nytimes.com/imagepages/2007/04/01/arts/01macaCA02ready.html. consulté : 1er mars 2017

9 Photographie extraite du site: https://www.metoperashop. org/shop/romeo-juliet-royal-ballet-dvd-3646. consulté : 1er mars 2017. 
J'ai pu observer que la corpulence des deux danseuses n'était pas la même : en 1984 le « corps classique " a changé et la maigreur faisait déjà office de canon dans le monde du ballet académique. Or, une analyse détaillée des portés montre que la plus mince des danseuses n'est cependant pas la plus légère... Dans la scène de la rencontre (scène du bal, version 1984) Roméo-Eagling soulève assez peu sa partenaire Juliette-Ferri ; on peut remarquer un léger à-coup à chaque fois qu'il la décolle du sol, et le plan final nous le montre légèrement essoufflé. Comment est-il donc possible qu'une danseuse aussi menue qu'Alessandra Ferri soit apparemment aussi lourde à porter ? De fait, avant la prise d'envol, Juliette-Fonteyn (version 1965), elle, est déjà suspendue au ciel par le regard, une dynamique de tension vers peut définir son attitude envers l'espace. L'appui du sol offert par Roméo-Nouréev ne fait qu'augmenter l'envergure de son envol. Alessandra Ferri, en revanche, garde les yeux baissés vers le sol, semblant ne pas vouloir quitter son lieu de prédilection. Alors même que Wayne Eagling tracte son bassin vers le haut celui-ci reste dynamiquement attiré vers le bas : le soulever se fait donc arrachement. La danseuse la plus fluette semble être la plus lourde et vice versa... Le « poids » n'a donc pas seulement à voir avec la corpulence mais plus fondamentalement avec l'organisation des forces, ainsi que vous l'illustre cette sculpture de Botero.
Figure 3 - Homme qui marche de Fernando Botero

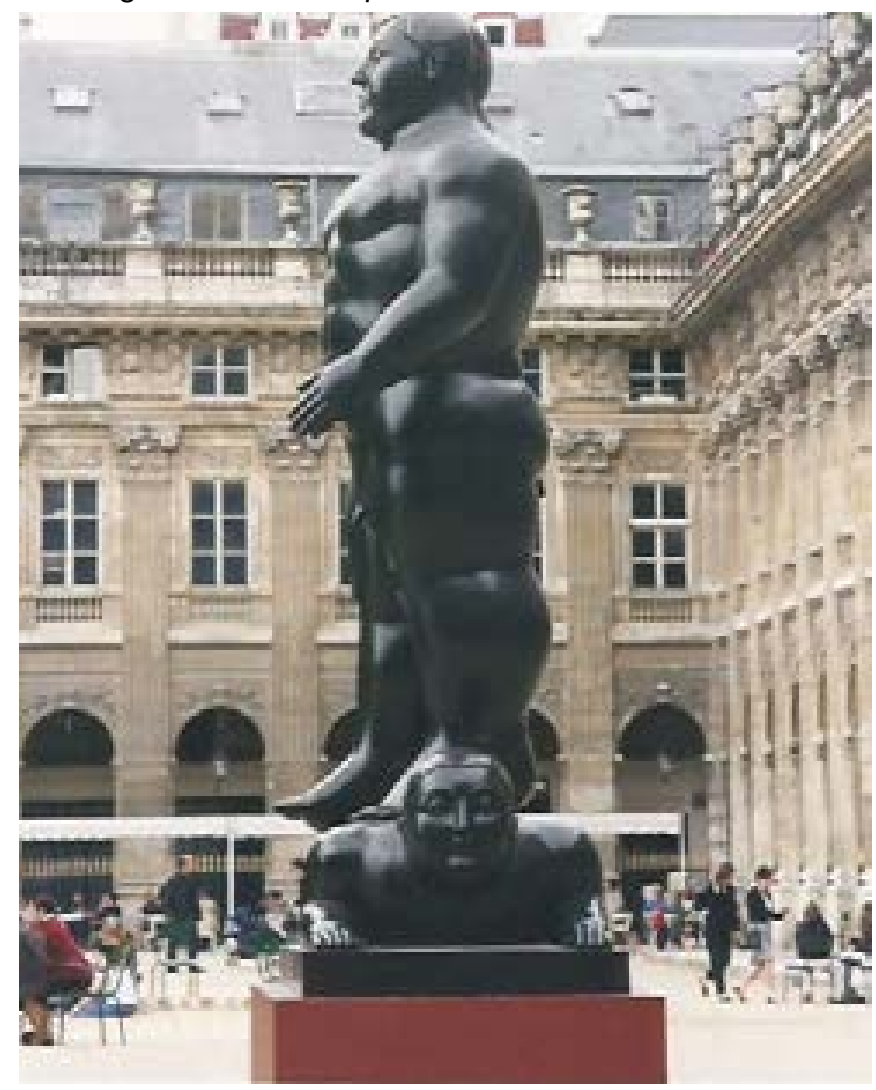

Source : Roquet

Pour qui a vu se mouvoir doucement mais gracieusement Elsa Wolliaston, je ne raconte rien d'étrange... II conviendrait alors de traduire weight, paramètre du système de l'Effort labanien, par « force motrice pondérale » ou plus simplement par « force ».

Jeu de flux

Une autre objection pourrait m'être opposée : « tout de même la danse, cela concerne bien des corps et surtout des corps jeunes ». Mais, concernant cette remarque, tout dépend de savoir s'il s'agit de pouvoir se montrer aux autres -en France, ne dit-on pas « se donner en spectacle » à propos de quelqu'un dont la tenue ou le geste est supposé déranger les autres ? - ou bien s'il s'agit de se poser la question 
de comment ça bouge. Je voudrais que nous regardions ensemble Tete Rusconi et Silvia Ceriani danser le tango $\operatorname{argentin}^{10}$, il ne s'agit là ni d'un homme jeune ni d'un corps d'athlète, et pourtant quelle danse... Les jeux de flux, le dialogue tonique engagé entre les protagonistes, ce qu'en reçoit le spectateur en son intimité (même devant un écran d'ordinateur) tout cela ne fait-il pas vaciller nos présupposés sur le « corps » du danseur?

\section{Désigner l'espace}

II n'y a plus de firmament ${ }^{11}$. Nous sommes à Paris, au Théâtre de la Ville, « temple de la danse contemporaine ». Dos au public, devant une salle pleine, Jean Babilée, danseur célébrissime du ballet classique, est assis, tout simplement, en silence. II ne nous regarde pas, il ne bouge pas, le temps semble suspendu... Le halo de sa «présence » (comme l'on dit en danse) se diffuse, atteint le public qui hésite entre expectative respectueuse et fascination, le Rien et le Tout en une même posture. Puis, doucement, en un geste à la simplicité émouvante, toujours de dos, le vieux danseur lève lentement le bras et désigne l'espace côté cour. Alors un monde s'ouvre... Qui a vu Jean Babilée, en son grand âge, en ouverture de la pièce de Joseph Nadj évoquée ici, ou qui a vu Kazuo Ono danser $L a$ Argentina, trouvera là encore que je ne raconte rien d'étrange, c'est le geste qui fait la danse, le si fameux « corps » n'a pas tant d'importance...

10 https://www.youtube.com/watch?v=XJDowJl0-7s. Consulté : 5 mai 2017.

11 Dernière apparition de Jean Babilée dans cette pièce de Joseph Nadj, en 2003, au Théâtre de la Ville à Paris.

\section{C'est le geste qui fabrique le corps}

Lorsque nous employons ce terme de « corps ", nous souscrivons au postulat implicite de la pensée occidentale qui définit le corps comme entité matérielle anatomique descriptible et univoque, dans son opposition à l'esprit ou à l'âme. Or, l'usage de ce signe linguistique est réducteur et ne rend pas compte de la dimension matérielle et sensible de notre vécu. C'est pour évacuer la fausse évidence portée par le concept de corps, que le philosophe Michel Bernard, à la suite des phénoménologues, a proposé de faire usage du concept de corporéité. Toute corporéité peut être envisagée à partir du fonctionnement intrinsèque du sentir : il ne s'agit plus ici de considérer une entité autonome et cernable qui reçoit/émet des informations et qui serait " le corps ", mais la concrétion d'un processus mouvant et complexe, celui du sentir. Ce qui continuerait alors par la suite à être nommé « le corps », ne saurait être envisagé que comme la matérialisation provisoire et visible, l'émergence d'un feu croisé de forces, de dynamiques (pulsionnelles, historiques, culturelles et sociales) qui traversent le dit « corps » (anatomique), non sans y laisser de traces. [RADIOS de la hanche].

Figure 4 : Radiographie de la hanche d'un bébé.

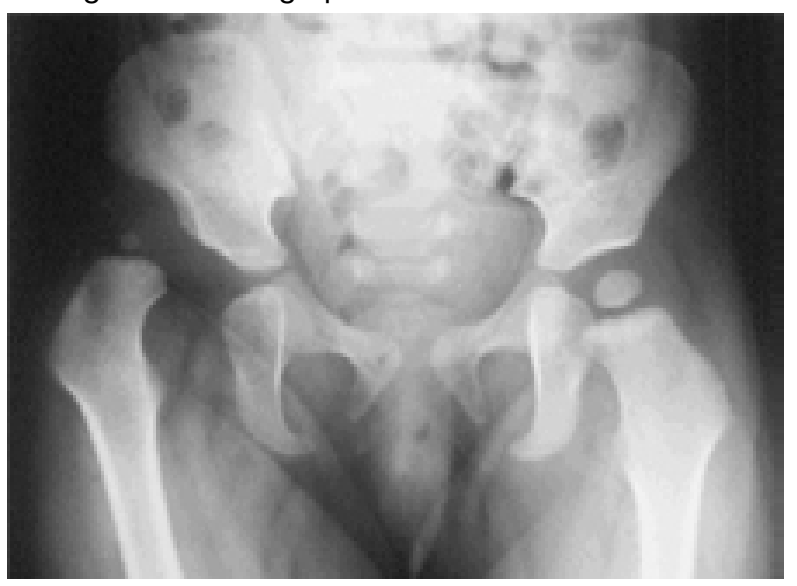

Source : Documentation personnelle. 
Figure 5 - Radiographie de la hanche d'un adulte.

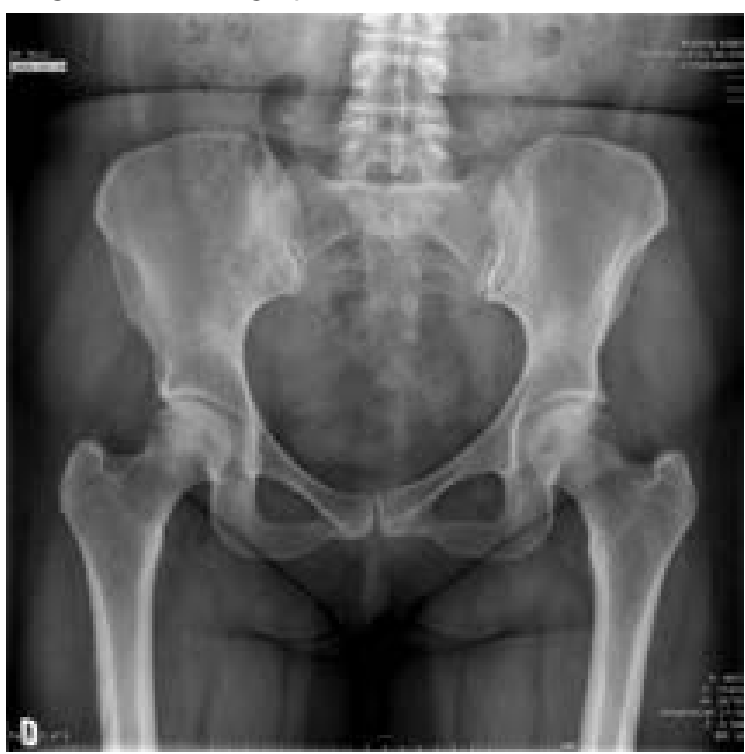

Source : Documentation personnelle.

C'est une manière singulière d'agir-percevoir au sein d'un environnement (mais faite de mille manières) qui va façonner une corporéité. Aussi il ne saurait y avoir de corporéité « en soi » mais seulement de multiples phénomènes de corporéités, chacune se définissant par un certain usage de soi. Parmi les travailleurs du corps, si certaines configurations corporelles semblent plus ou moins cousines, c'est bien souvent que ces travailleurs et travailleuses partagent le même geste, c'est-à-dire, la même pratique : le même apprentissage, le même entraînement, le même idéal.

Figure 6 - Haïle Gebreselassie, champion marathon.

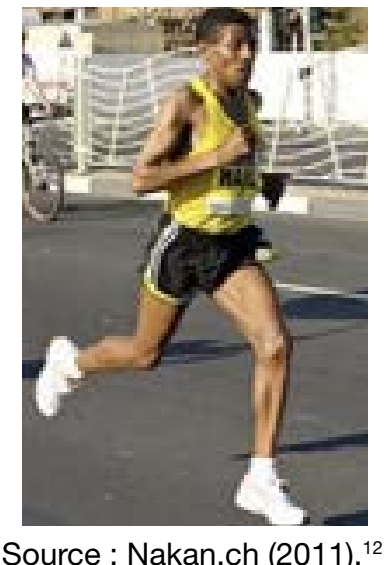

12 Photographieextraitedu site:https://www.nakan.ch/wp/2011/09/19/ la-bonne-posture-en-course-a-pied/. Consulté : 1er mars 2017
Figure 7 - Christine Ohuruogu, campeã dos 400 metros.

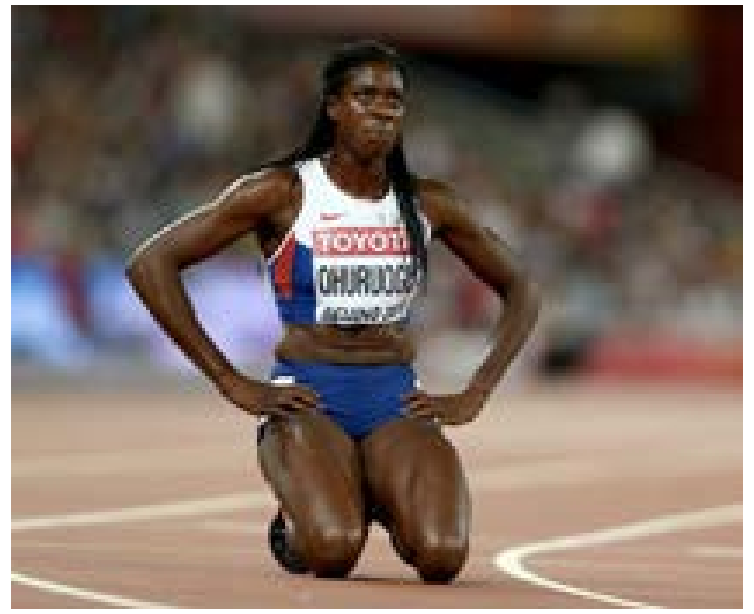

Source: Zimbio (2015). ${ }^{13}$

Comme le dit très justement Hubert Godard, "c'est le geste qui fabrique le corps".$^{14}$

\section{— Les quatre structures de la corporéité}

Chez Godard, comme chez Laban, c'est l'agir-percevoir, la manière dont cet agir-percevoir se produit, qui importent en premier lieu. Dans ce contexte donc le terme de « structure " n'en réfère pas au sens commun qui visualisera ici le plus souvent une ossature circonscrite et immuable. Le terme, synonyme ici de système, désigne un ensemble organisé de rapports entre divers éléments ${ }^{15}$. Le concept de corporéité désigne donc le fonctionnement d'un système de systèmes, une « superstructure » en quelque sorte. Comme nous avons

13 Photographie extraite du site: http://www.zimbio.com/pictures/2fxeeXS5n4T/15th+|AAF+World+Athletics+Championships+Beijing/MtwDWR8s-Wa/Christine+Ohuruogu.

Consulté : 1er mars 2017

14 Hubert Godard. "Le geste manquant », IO, Revue de psychanalyse, n. 5, Ramonville St Agne, ERES, 1994, p. 73.

15 Plus qu'au sens courant du terme, le terme de " structure » fait référence chez Godard davantage au sens mathématique du terme (comme système de relations existant entre diverses théories mathématiques. Voir le groupe Bourbaki auquel appartenait André Weil qui a travaillé avec C. Lévi-Strauss sur Les structures élémentaires de la parenté). 
développé les enjeux pratiques de cette théorisation (née elle-même de la pratique) lors des ateliers, je ferai un simple rappel de ces quatre structures, à l'aide d'un exemple, celui du dos.

a) La structure somatique ou « le mal de dos, le mal du siècle ".

La structure somatique concerne l'anatomie et la physiologie traditionnelles. Pour l'anatomiste, le dos c'est une charpente osseuse (la colonne vertébrale), des muscles (les muscles postérieurs du tronc), des nerfs, des vaisseaux etc. Pour le kinésithérapeute soulager un mal de dos (une contracture musculaire douloureuse, par exemple) c'est, par l'intermédiaire de massages et d'exercices, tenter de traiter un désordre mécanique considéré comme local.

b) la structure coordinative ou " sauter avec son dos ».

Comme son nom l'indique la structure coordinative concerne les coordinations. Pour le dire simplement, une coordination est la manière, tant spatiale que temporelle, dont s'organisent, s'associent entre elles les parties du corps lors de tel ou tel mouvement. La structure coordinative intéresse particulièrement les psychomotriciens, par exemple ceux travaillant dans le domaine de l'éducation fonctionnelle des enfants porteurs de handicaps moteurs. Cette coopération de systèmes (synergies musculaires et nerveuses) est un automatisme (moteur et perceptif) acquis. Si plier les deux jambes pour sauter est une coordination, sauter avec son dos invite à observer plus précisément la relation tonique entre les membres et l'axe central lors de tel ou tel saut.

c) La structure perceptive ou « sentir l'air sur son dos ».

Le travail sur la structure perceptive intéresse entre autres les rééducateurs d'enfants porteurs de handicaps sensoriels ainsi que tous ceux qui proposent des activités d'éveil perceptif et moteur. En Occident, la toute-puissance culturelle de l'image (dans le miroir, sur un écran, sur le papier) entraîne l'individu à se percevoir lui-même en deux dimensions. En atelier, on peut être amené à bouger en " sentant l'air sur son dos » : cette métaphore peut ouvrir un nouvel accès à une autre perception (de l'espace arrière, de l'autre) par le recours à l'imaginaire et permettre alors de marcher plus aisément à reculons, par exemple.

d) La structure psychique (ou structure symbolique) ou « en avoir plein le dos ».

L'aspect symbolique d'un geste désigne ici comment sa relation au contexte le rend porteur d'un sens. La structure symbolique concerne le sens du geste en général, ce sens n'étant pas circonscrit uniquement à la « signification ». C'est la différence en français quand je dis « cela a telle signification » ou bien « cela fait sens ». La vie du dos est en relation avec la vie psychique de l'individu; ce que soulignait Boris Dolto dans le chapitre IX de son livre Le corps entre les mains ${ }^{16}$ intitulé : «physiologie, psychisme et lombalgies ».

Imaginons, pour finir, cette situation : un dialogue imaginaire entre amoureux lors duquel l'un des partenaires dit à l'autre : j'aime ton corps. De quoi parle-t-il (ou elle) ? D'une partie de son anatomie, de sa morphologie (structure somatique) ? D'une manière de bouger ou de se tenir qui lui plait chez l'autre (structure coordinative) ? D'une façon d'accueillir les sensations tactiles (structure perceptive) ? De son engagement dans la conversation amoureuse, des paroles prononcées (le sens des mots) ? Vous voyez que cet outil peut nous aider à y voir plus clair lorsque nous observons des compor-

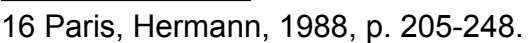


tements ou des discours, alors que le terme de « corps " ne nous y aide pas. Mais vous me direz, le concept de « corporéité » n'est pas si aisé à utiliser dans le langage courant... II ne $s^{\prime}$ agit pas de le bannir ${ }^{17}$ mais je vous enjoins à penser en termes de geste plutôt qu'en termes de corps.

\section{Lire le geste, un outil pour la} recherche en danse

Vous avez compris que, pour Hubert et moi-même, c'est au sein du geste lui-même que réside le sens (multiple) de la danse. Plutôt que d'aligner encore des propos théoriques je vais tenter de démontrer à l'aide d'un exemple que lire le geste dansé est nécessaire pour ouvrir les possibles des regards portés sur la danse. Pour rester dans le fil de ce que nous avons déjà vu en atelier, je vais organiser la réflexion autour d'un exemple pris dans la comédie musicale (musical), celui de The band wagon ${ }^{18}$, film de Vincente Minelli (1953), joué et dansé par Fred Astaire et Cyd Charisse. Une première question se pose à nous : à quel(s) problème(s) chercherait à répondre l'analyse de l'œuvre ? Quelle(s) question(s) pose mon regard ? Faisons par exemple l'hypothèse que nous avons l'intuition d'un lien esthétique entre l'art de filmer de Minelli et l'art de danser de Fred Astaire et nous irons alors explorer cette hypothèse. Pourquoi lire le geste des protagonistes de cette comédie musicale ? N'y a-t-il pas déjà moult études sur ce genre cinématographique ? Un constat s'impose : les classiques de la co-

17 La recherche et son " chemin désirant » (étymologiquement quaerere) est bien ce qui s'oppose à toute police de la pensée...

18 Tous en scène, pour la version française. médie musicale hollywoodienne ont bénéficié de nombreuses études proposant parfois des notions novatrices et soucieuses de prendre en compte le rôle spécifique qu'y joue la musique $^{19}$ mais sans s'occuper du geste dansé. Comment peut-on analyser les relations musique/image dans une comédie musicale sans prendre en compte le fait que ces deux éléments entrent eux-mêmes en relation avec les corps en mouvement ? II existe une véritable carence d'analyse de ce que font les danseurs, ce qui motive donc notre désir de le faire.

\section{Dancing in the dark}

The Band Wagon est un film réflexif articulant à la fois l'identité du ballet classique et du Music Hall. The Band Wagon est un film construit en danse ; un danseur est au coeur du récit et pas n'importe quel danseur : Fred Astaire qui, dit la doxa, symbolise à lui seul la comédie musicale américaine, [et] qui fut le danseur de claquettes le plus célèbre du monde ${ }^{20}$. Qu'avions-nous découvert, en atelier, avec l'analyse du duo Dancing in the dark extrait de ce film ? Au niveau de la narration, la scène de Dancing in the dark répondait à la question : " pouvons-nous danser ensemble ? » et posait les prémisses d'une histoire d'amour. Nous avons observé comment une certaine qualité de l'interaction reposait sur la spécificité de la couleur expressive de la danse des deux protagonistes et principalement sur la relation à l'espace de Fred Astaire. Fred est

19 Rick Altman La Comédie musicale hollywoodienne, Paris, A. Colin, 1992.

20 Fred Astaire, En revenant sur mes pas, Paris, F. Bourin, 1990, trad. de l'anglais par René Dor, 1ère éd. Step in time, N.Y. Harper \& Brothers, 1959, 4ème de couverture. 
le pivot des mouvements de l'éros charnel décliné par la danseuse ; il tient en quelque sorte l'espace tout au long du duo et Cyd Charisse alterne entre des moments où elle épouse cet espace et se repose sur son axe à lui (quand elle est tenue par son partenaire) et des moments de reprise où elle danse seule et affirme une succession de figures. Pour résumer, nous pourrions reprendre l'heureuse formule d'Hubert Godard : "Fred Astaire fait danser le paysage tandis que Cyd Charisse danse dans le paysage créé par le danseur ${ }^{21}$ ".

Tout cela est bel et bon me direz-vous, mais Fred Astaire est aussi chorégraphe, chanteur, auteur de chansons, comédien... II ne fait pas que danser en couple et quel peut être le rapport entre ce que nous observons dans ce duo et ce qu'il fait quand il est seul ? Je vais donc tenter de répondre à cette question en centrant l'analyse sur un " solo » de Fred Astaire, By myself. Je dis « solo » et pourtant ici personne ne danse...

\section{By myself}

By myself est le premier numéro du film The band wagon. Tony, danseur qui a connu son heure de gloire à Hollywood, descend du train en gare de New York, accueilli par des journalistes, il est vite dépité, car les journalistes sont venus pour Ava Gardner en personne et non pas pour lui. Mélancolique et seul, il chante By myself en déambulant le long du quai, chanson dont les paroles évoquent l'incertitude de l'avenir et la solitude du personnage. By myself est pour le personnage un moment d'introspection, il l'isole dans une bulle. Les autres personnes présentes sur le quai, pour la plupart des travailleurs de la gare en uniformes sombres, défilent à un rythme différent du sien, en général bien plus rapide, comme si le rythme de Tony, sa musique intérieure, suffisaient à l'isoler du reste de l'humanité industrielle et laborieuse du lieu. Ce numéro est chanté par Astaire lui-même et se veut non dansé aussi la caméra privilégie systématiquement le haut du corps. Le début commence comme une marche simple, suspendue entre les appuis, puis, à partir du kiosque, la marche se fait ternaire sur chaque appui, à l'occasion d'un petit rebond à chaque pas la suspension haute du corps de $F$. Astaire s'accentue, permettant la torsion profonde du dos, le balancé des épaules se fait plus prononcé, l'élasticité du pas augmente, la marche est devenue danse... Le swing du pas ne serait-il pas ce qui permet le fondu enchaîné entre marche ordinaire et marche dansée ?

La bulle d'espace de Fred Astaire (appelée aussi espace péri-personnel ou kinésphère) est un espace d'accueil, complètement ouvert, une partenaire imaginaire y est comme déjà invitée. Tout dans sa démarche nous laisse découvrir la mélodie cinétique d'un corps déjà réceptif, prêt à composer avec l'autre, dessinant ainsi en creux une figure de l'absence. En fait, pour saisir le rapport à l'espace de Fred Astaire il faut se souvenir qu'il n'a pas ou peu dansé seul. Depuis sa plus tendre enfance Astaire a dansé en partenariat (to partner en anglais) : " toutes mes danses se faisaient avec Adèle, probablement parce que nous formions un duo depuis toujours ${ }^{22}$ » et qui est Adèle ? Sa sœur chérie, avec qui l'entente était parfaite, selon ses dires. L'apprentissage des danses de

22 Fred Astaire, En revenant sur mes pas, op. cit., p. 142. 
ba/23 commence par la marche. Qu'a donc fait Astaire dès la plus tendre enfance ? Marcher et danser avec une autre, sa sœur bien aimée. On pourrait certainement dire que, concernant la danse, «Fred Astaire » seul, cela n'existe pas...

\section{De marche en danse}

Comme le souligne Rodowick ${ }^{24}$, dans les comédies musicales jouant du star système, le danseur ne se confond jamais avec le personnage et le public attend que le personnage révèle la star: même quand Astaire et Charisse ne dansent pas, les spectateurs, non seulement attendent la performance, mais continuent à voir non pas des personnages -et ce quelle que soit la qualité du jeu des comédiens- mais un danseur ou une danseuse jouant un personnage. Autrement dit, même en présence d'un jeu parfaitement réaliste, le spectateur persiste à voir la danse derrière le rôle. C'est même pour le plaisir de cette découverte qu'il va voir ces films... Chacun des gestes des personnages, même banal, est donc vu comme étant potentiellement un geste dansé. Cet effet est renforcé par l'utilisation de gestes quotidiens dans les chorégraphies: marcher ou monter un escalier peuvent devenir des éléments d'une chorégraphie, rendant ainsi extrêmement floues les frontières entre jeu rythmique et jeu réaliste. C'est donc dans la continuité avec les

23 Ball room dances, à traduire en français par « danses de bal » plutôt que par danses de salon.

24 « Dans les comédies musicales, le danseur ne se fond jamais dans le personnage, le public désire plutôt que le personnage permette l'expression profonde de l'interprète ou bien que le personnage révèle l'Etoile (la star) que nous avons toujours connue. " David Norman Rodowick, « From An American in Paris : Identity and desire in two musicals by Vincente Minnelli » dans Comédie musicale : les jeux du désir. De l'âge d'or aux réminiscences, dir. par S. Chalaye et $\mathrm{G}$. Mouëllic, Presses Universitaires de Rennes, 2008, Rennes, p.48. gestes ordinaires, et non pas dans la césure, que doivent se comprendre et s'interpréter les gestes dansés. Lors de l'altercation avec le metteur en scène durant laquelle il quitte la répétition pour reprendre son indépendance, Tony Hunter, en colère s'écrit :

« I am not Nijinski, I am not Marlon Brando, I am Mrs Hunter's little boy, Tony, song and dance man ». (Je ne suis pas Nijinski / Je ne suis pas Marlon Brando / Je suis le petit garçon de Mme Hunter, Tony, un homme qui chante et qui danse!). Ne pourrions-nous pas crier tous à notre tour : nous ne sommes pas des " corps ", nous ne sommes pas des images, nous sommes de simples humains, qui, chacun à leur façon, perçoivent et bougent... qu'on appelle ou non cela de la « danse »! ?

Rêve et réalité

L'analyse détaillée de Dancing in the dark et de By myself ${ }^{25}$ permet de tisser du liant entre l'esthétique du geste astairien et celle du cinéaste. En accordant ensemble l'aisance du geste et l'aisance de la caméra ; en liant en un ruban de moebius ${ }^{26}$ les fluidités des mouvements de caméra et de la danse ; en évitant les aspérités, ruptures ou disjonctions ; en proposant une manière singulière de faire plutôt qu'une recette de l'entertainment déjà prête ; en voilant l'habileté derrière une simplicité apparente, les artistes, danseurs et cinéaste, partagent une même esthétique qui est celle de l'élégance. Mais la particularité de ce solo d'ouverture et l'analyse du geste de marcher

25 Cf. conférence Christine Roquet et Cécile Sorin, Semaine des Arts, Université Paris 8, http://www-artweb.univ-paris8. fr/?The-Band-Wagon.

26 Ou leminscate. 
dans l'intégralité du film ne nous amèneraien$\mathrm{t}$-ils pas à nous interroger sur les relations entre trivialité du quotidien et imaginaire du merveilleux au sein de l'œuvre de Minelli ? Le film date de 1953, historiquement ces années 5060 voient la disparition de la comédie musicale et la concurrence de la télévision ; on quittera alors, d'une certaine manière, le monde du rêve. En quoi le geste de Fred Astaire, jouant ici un danseur en fin de carrière donc en passe de quitter le monde qui était le sien jusqu'alors, en quoi ce geste dansé interroge-t-il chacun sur son propre «monde du rêve »? Nous partageons avec Isabelle Ginot cette idée " qu'un rêve n'est jamais fini d'interpréter ${ }^{27}$ »; pour qu'il en soit de même pour un film ou une chorégraphie ne faut-il pas laisser flotter notre attention ? Epouser les méandres du mouvement ? Suivre les traces des gestes? Se laisser bercer des mélodies cinétiques ?

Chez Fred Astaire, c'est dans une sorte de fondu envers l'espace (et non dans un fondu dans le sol) que s'origine une danse tout en legato. La danseuse Constance Vallis Hill nomme aussi le style de Fred Astaire de « soft-shoe tap dance $^{28} \gg$; intraduisible, cette appellation tente pourtant de nommer quelque chose d'une qualité spécifique du tactile dans le rapport au sol, et si Astaire caresse le sol c'est que l'espace le porte... La lecture de cette attitude envers l'espace, celle des modes d'anticipation du geste, le suivi empathique du flux du mouve-

27 Dossier d'HDR, La critique en danse contemporaine : théories et pratique, pertinences et délires, sous la dir. de J.P. Olive, Paris 8, Sept. 2006 (publication en cours)

28 Constance Valis Hill, Tap dancing america, USA, Oxford Univ. Press, 2010, p. 113. Soft shoe fait référence à la danse en chaussures ordinaires, danse issue des " sand dances" où l'on glisse en chaussures sur du sable. Le tap dance, de son côté, se danse en chaussures ferrées, le geste de frapper est donc plus présent que celui de glisser que l'on trouvera davantage dans la technique soft shoe. Je remercie Stephan Cotell de m'avoir précisé cela. ment, l'observation des modulations toniques, etc. Vous l'avez compris, la lecture du geste dansé a pour dessein d'ouvrir le regard porté sur les œuvres. De concert avec d'autres perspectives (analyse musicale ou cinématographique ici) elle s'en vient nourrir les perceptions et interrogations indécises et flottantes du spectacteur $^{29}$, pour faire de notre regard une danse, sensible à celle que l'interprète nous offre...

Référence

ALTAMAN, Rick. La comédie musicale hollywoodienne. Paris: A. Colin, 1992.

ASTAIRE, Fred. En revenant sur mes pas. Paris: F. Bourin, 1990, Trad. de l'anglais par René Dor, 1ère éd. Step in time, New York: Harper \& Brothers, 1959.

DOLTO, Boris. Physiologie, psychisme et lombalgies. In: Le corps entre les mains. Paris: Hermann, 1988. p. 205-248.

GODART, Hubert. Le geste et sa perception. In: GINOT, I.; MICHEL, M. La danse au XXème siècle. Paris: Bordas, 1995.

de psychanalyse, n. 5, Ramonville St. Agne, ERES, 1994.

MET OPERA SHOP. Romeo \& Juliet - Royal Ballet (DVD). Kultur: [London]: 2017.

Disponible en: https://www.metoperashop. org/shop/romeo-juliet-royal-ballet-dvd-3646. Consulté le 1er mars. 2017.

29 Pavis P., L'analyse des spectacles, Paris, Nathan, 1996, p. 120. 
PAVIS, Patrice. L'analyse des spectacles. Paris, Nathan, 1996

THE BABITT AND THE BROMIDE In: Ziegfled follies, film de Vicente Minelli, 1946.

https: / / w w w.youtube.com / watch?V=IMKbGRCbsaw. Consulté: 1er mars 2017.

Valis Hill Constance, Tap dancing america, USA, Oxford Univ. Press, 2010

Recebido: 29/05/2017 Aprovado: 08/07/2017 